\title{
PREVALENCE AND HISTOPATHOLOGICAL EFFECTS OF SCHISTOSOMA HAEMATOBIUM ON THE FEMALE GENITAL TRACT IN SOHAG GOVERNORATE, UPPER EGYPT \\ By \\ NOHA S. AHMED ${ }^{1}$, SHEREN F. MAHMOUD ${ }^{2}$, AHMED A.A TAHA ${ }^{3}$, NAHED A. EL-OSSILY ${ }^{4}$, and YASSER M. MOHAMED ${ }^{4}$ \\ Department of Medical Parasitology ${ }^{1,4}$, Department of Pathology ${ }^{2}$, and Department of Obstetrics and Gynaecology ${ }^{3}$, Faculty of Medicine, Sohag University ${ }^{1,2,3}$, and Faculty of Medicine, Assiut University ${ }^{4}$, Egypt \\ ( ${ }^{*}$ Correspondence: yasser_mokh@yahoo.com) \\ Abstract
}

Urinary schistosomiasis is a chronic granulomatous inflammation affects many body systems including female genital tract. The present study determined the prevalence and infection intensity of urogenital schistosomiasis and made a retrospective analysis of histopathological changes in female genital tract affected by $S$. haematobium among female patients attended Sohag University Hospitals, from October 2016 to September 2019. A total of 100 fallopian tube, 50 cervical and 50 vaginal specimens were collected from 200 female patients (126 rural \& 74 urban) aged between 20-50 years; suffering from infertility, bleeding, pelvic discomfort and or dyspareunia. Histopathological examination of tissue biopsies was performed. Urine examination for all participants was done to detect S. haematobium eggs and infection intensity by using a standard urine filtration technique. Seven specimens $(7 \%)$ were documented to have fallopian tube, five (10\%) cervical and two (4\%) vaginal schistosomiasis. Twelve out of 14 genital schistosomiasis cases were rural $(85.7 \%)$ and two cases were urban (14.3\%). Urine examination revealed 18 Schistosoma haematobium egg positive samples out of 200 participants $(9 \%),\{6(33.3 \%)$ with light infection intensity and $12(66.7 \%)$ with heavy infection intensity $\}$. Nine out of 14 female genital schistosomiasis (FGS) cases (64.2\%) were positive for Schistosoma haematobium eggs in urine, eight (88.8\%) of them with heavy infection intensity.

Key words: S. haematobium, prevalence, Intensity, FGS, Sohag Governorate, Egypt

\section{Introduction}

Schistosomiasis is a complicated long standing chronic disease with serious morbidity worldwide (Gouveia et al, 2019). This water-dependent disease is endemic in rural areas (Senghor et al, 2014). The prevalence of active and past infection with urogenital schistosomiasis is high in rural than urban areas, with a lack of drinking water and the daily water contact activities undertaken in rural areas, such as swimming, bathing, washing clothes and fishing (Helling-Giese et al, 1996; Senghor et al, 2014; Ekpo et al, 2017).

Female genital schistosomiasis (FGS) is a gynecological disease due to Schistosoma haematobium infection; it is endemic throughout Africa with a major burden of disease in sub-Saharan Africa (Steinmann et al, 2006; Colley et al, 2014). It is related to poverty by poor sanitation and dependency on contaminated water sources as rivers, dams, and lakes. Populations at risk have limited access to adequate health care (Hotez and Kamath, 2009).

Vaginal schistosomiasis was reported for the first time in Egypt in 1899 (Madd-en, 1899). Clinically apparent vulval, vaginal and/or cervical schistosomiasis has been reported from both endemic and non-endemic areas. This led the Gender Task Force of the WHO Tropical Disease Research Program to include FGS in a list of scientific areas that deserve high research priority (Vlassoff, 1997). The most pervasive form of urogenital schistosomiasis is caused by $S$. haematobium predominant in Africa and Middle East (WHO, 2015). Cervix, fallopian tubes, and vagina are the commonest gynecological sites harboring $S$. haematobium (Kjetland et al, 2012).

The lesions associated with FGS are relat- 
ed to the parasite's deposition of eggs in the genital mucosa (Poggensee and Feldmeier, 2001). They may appear as singular grains, grains in clusters, and as homogenous, yellow areas (Norseth et al, 2014). Besides, characteristic blood vessels in the cervical mucosa are an important sign of genital schistosomiasis (Kjetland et al, 2005). FGS may create lesions and inflammation in female genital tract, causing bleeding, pelvic discomfort, dyspareunia, and infertility (Kjetland et al, 2006). So, there is a growing concern that it may increase the risk of acquiring human immunodeficiency virus (HIV) (Mbabazi et al, 2011; Kjetland et al, 2012; Mbah et al, 2013). The relationship between FGS and HIV was explored in two independent cross-sectional studies including 1,000 women, showed that those with urogenital schistosomiasis have a higher prevalence of HIV (Downs et al, 2011). The public health importance of FGS is not fully recognized nor is its control adequately addressed (Christinet et al, 2016).

The present study was done to make a retrospective analysis of histopathological changes in the female genital tract affected by Schistosoma haematobium with determination of the prevalence and infection intensity of urogenital schistosomiasis among female patients attending Sohag University Hospital, Sohag Governorate.

\section{Materials and Methods}

This retrospective analytical study was done in the Research Laboratory of Medical Parasitology and Pathology Departments, Faculty of Medicine, Sohag University from October 2016 to September 2019.

Collection of tissue samples: A total of 100 fallopian tube, 50 cervical and 50 vaginal specimens were collected from 200 female patients (126 rural and 74 urban), aged between 20-50 years, with the mean age (32.7 \pm 7.3 years). They were divided into 2 age groups: GI: 108 cases aged $20-<35$ years and GII: 92 cases aged 35-50 years. They suffered from infertility, bleeding, pelvic discomfort and/or dyspareunia. All partici- pants in this study had non-processed fresh water contact at some point in their lives.

All the biopsies referred from Obstetrics and Gynecology Department were histopathologically examined for effect of Schistosoma haematobium on their genital tract.

Histopathological examination: Specimens were fixed in $10 \%$ formalin, paraffin embedded and sections of $5 \mu \mathrm{m}$ thickness using standard techniques (Culling, 1993). Sections were stained with Hematoxylin and Eosin, mounted in DPX, and examined using compound microscope.

Diagnosis of urogenital schistosomiasis, $10 \mathrm{~mL}$ urine sample was collected from each patient between 10 a.m. \& 2 p.m, the peak daily egg excretion time (Doehring, 1985). Microscopic examination of each urine sample for S. haematobium eggs and infection intensity was performed (Senghor et al, 2014). 10ml urine was passed via a Millipore filter $(12 \mu \mathrm{m}$ polycarbonate filter), and this filter was examined with a 10x objective. Number of eggs per filter was counted, and the infection intensity was classified as light $(<50$ eggs $/ 10 \mathrm{ml}$ of urine) or heavy $(\geq 50$ eggs/10ml of urine), after WHO (2002).

The research system followed the ethical standards of confidentiality and freedom to participate. They were informed about the aim and assured that their privacy would be protected and all gave a written consent for studying their specimens.

\section{Results}

Histopathological examination of all biopsies showed that seven specimens (7\%) were documented to have fallopian tube, five $(10 \%)$ cervical and two (4\%) vaginal schistosomiasis, (with a total percentage 14/200 or $7 \%$ ) as documented by the Medical Parasitology and Pathology Departments, Faculty of Medicine, Sohag University. Twelve out of 14 genital schistosomiasis cases were rural $(85.7 \%)$ and two cases were urban $(14.3 \%)$. Of the 14 FGS cases $9(64.2 \%)$ were in GI and $5(35.8 \%)$ were in GII (Tab. 1).

Serial sections from the examined biopsies 
showed deposits of multiple degenerated and calcified bilharzial eggs surrounded by granulomatous reaction in the form of chronic inflammatory cells rich in macrophages and eosinophils in addition to lymphocytes, plasma cells, foreign body giant cells and fibroblasts with surrounding variable degrees of fibrosis within the subepithelial and submucosal layers (Figs. 1-8). All specimens did not show malignant or premalignant cells. Of 200 urine samples examined for $S$. haematobium eggs, 18 (9\%) were infected, with egg mean geometric count (EMGC) of $65 \mathrm{eggs} / 10 \mathrm{ml}$ of urine, $\{6(33.3 \%)$ with light infection intensity and $12(66.7 \%)$ with heavy infection intensity . Of the $18 \mathrm{~S}$. haematobium cases $13(72.2 \%)$ were in GI and $5(27.8 \%)$ were in GII (Tab. 2). Nine out of the 14 genital schistosomiasis cases $(64.2 \%)$ were positive for $S$. haematobium eggs in urine $8(88.8 \%)$ with heavy infection intensity \& $1(11.2 \%)$ with light infection intensity (Tab. 3). All FGS cases with $S$. haematobium heavy infection intensity were rural in GI.

Statistical analysis: Data were expressed as a percentage, and analysis was carried out using Chi square test (x2). Probability P. value $<0.05$ was considered significant.

Table 1: Correlation between prevalence of FGS and participant age group.

\begin{tabular}{|c|c|c|c|c|c|c|}
\hline \multirow{2}{*}{ Age group } & \multicolumn{5}{|c|}{ No. of FGS cases } & \multirow{2}{*}{ P. value } \\
\cline { 2 - 5 } & Rural & Urban & No. & $\%$ & & \\
\hline GI $(20-<35 \mathrm{y})$ & 8 & 1 & 9 & 64 & \multirow{2}{*}{1.218} & 0.269 \\
\hline GII (35-50y) & 4 & 1 & 5 & 36 & & \\
\hline Total & 12 & 2 & 14 & & & \\
\hline
\end{tabular}

Prevalence of FGS was higher in younger (GI) than GII, without significant $(\mathrm{P}=0.269)$.

Table 2: Correlation between participant age group and prevalence \& infection intensity of urogenital schistosomiasis.

\begin{tabular}{|c|c|c|c|c|c|c|c|c|c|}
\hline \multirow{3}{*}{ Age group } & \multirow{3}{*}{ Rural } & \multirow{3}{*}{ Urban } & \multirow{3}{*}{ Total } & \multicolumn{2}{|c|}{ Positive $S$. haematobiam eggs } & \multicolumn{4}{|c|}{ Infection intensity } \\
\hline & & & & \multirow{2}{*}{ No. } & \multirow{2}{*}{$\%$} & \multicolumn{2}{|c|}{ Light } & \multicolumn{2}{|c|}{ Heavy } \\
\hline & & & & & & No. & $\%$ & No. & $\%$ \\
\hline GI $(20-<35 y)$ & 67 & 41 & 108 & 13 & 72 & 3 & 50 & 10 & 83 \\
\hline GII (35-50y) & 59 & 33 & 92 & 5 & 28 & 3 & 50 & 2 & 17 \\
\hline Total & 126 & 74 & 200 & 18 & & 6 & & 12 & \\
\hline $\mathrm{X}^{2}$ & & & & \multicolumn{2}{|c|}{5.321} & & & \multicolumn{2}{|c|}{7.981} \\
\hline P. value & & & & \multicolumn{2}{|c|}{$0.021 *$} & & & \multicolumn{2}{|c|}{$0.005 *$} \\
\hline
\end{tabular}

Prevalence and infection intensity of uro- higher in GI than GII ( $\mathrm{P}=0.021 \& 0.005$ regenital schistosomiasis were significantly spectively).

Table 3: Correlation between prevalence of FGS and S.haematobium infection intensity.

\begin{tabular}{|c|c|c|c|c|c|c|c|c|}
\hline \multirow{3}{*}{ Urine } & \multirow{2}{*}{\multicolumn{2}{|c|}{$\begin{array}{c}\text { FGS } \\
\text { Total no. }=14\end{array}$}} & \multicolumn{4}{|c|}{ S. haematobium infection intensity } & \multirow{3}{*}{$X^{2}$} & \multirow{3}{*}{ P. value } \\
\hline & & & \multicolumn{2}{|c|}{ Light } & \multicolumn{2}{|c|}{ Heavy } & & \\
\hline & No. & $\%$ & No. & $\%$ & No. & $\%$ & & \\
\hline FGS with $S$. haematobium eggs & 9 & 64 & 1 & 7 & 8 & 57 & \multirow{2}{*}{5.9} & \multirow{2}{*}{$0.015^{*}$} \\
\hline FGS without $S$. haematobium eggs & 5 & 36 & - & - & - & - & & \\
\hline
\end{tabular}

Prevalence of FGS was significantly correlated with $S$. haematobium infection intensity $(\mathrm{P}=$ $0.015)$.

\section{Discussion}

Female genital schistosomiasis (FGS) is incredibly common. Approximately two-thirds of Africa's 200 million schistosomiasis cases are caused by $S$. haematobium, and it is estimated that up to three-quarters of girls and women with $S$. haematobium infection have FGS (Hotez et al, 2019). Genital tissue biopsy with histopathological examination was deemed to be the gold standard for diagnosis of FGS (Kjetland et al, 2012).
In the present study, the prevalence of FGS in all examined cases was $(14 / 200=$ $7 \%$ ). Several local and international studies reported different FGS prevalence rates in different countries. Talaat et al, (2004) in Egypt identified female genital schistosomiasis found prevalence of FGS 33\%. Screening studies targeting the general population in endemic regions for schistosomiasis as Madagascar, Tanzania, Malawi and Niger, FGS prevalence ranged from $33 \%$ in Mada- 
gascar to $75 \%$ in Niger, with Tanzania and Malawi in between (Renaud et al, 1989; Leutscher et al, 1998; Poggensee et al, 2000; Kjetland et al, 2005). Hotez and Whitham, (2014) reported that the overall prevalence of FGS in about 85 million women infected with urogenital schistosomiasis, of whom one-third suffered from FGS.

In the present study, the lower prevalence of FGS can be explained by the use of successful schistosomiasis prevention and control programs in Egypt with mass drug administration (MDA) with the antiparasitic drug, praziquantel.

In the present study, 12/14 genital schistosomiasis cases were rural $(85.7 \%)$ and two cases were urban (14.3\%). This may be due to the fact that, the prevalence of active and past infection with urogenital schistosomiasis as well as infection intensity were much higher in rural areas with lack of drinking water for human daily use. This agreed with Helling-Giese et al. (1996); Senghor et al. (2014); Galappaththi-Arachchige et al. (2016), Ekpo et al. (2017).

The infection intensity of the urogenital schistosomiasis influenced the FGS risk, and there was a considerable increase in risk between cases with light and heavy infections (Poggensee et al, 2000). In the present study, the prevalence of FGS was significantly correlated with $S$. haematobium infection intensity $(\mathrm{P}=0.015)$. This agreed with Ekpo et al. (2017) and Shukla et al. (2019).

In the present study, of 14 FGS cases 9 $(64 \%)$ were in GI and $5(36 \%)$ were in GII, but without significant $(\mathrm{P}=0.269)$. This finding was attributable to intense water contact activities resulting in higher prevalence and infection intensity of urogenital schistosomiasis in younger females (GI). This fact was more or less in accordance with other authors (Hegertun et al, 2013; Senghor et al, 2014; Hotez et al, 2019; Shukla et al, 2019).

In this study, the cervix was the most prevalent site $(5 / 50=10 \%)$, followed by fallopian tube (7\%) and lastly the vagina (4\%). This agreed with Kjetland et al. (2012) and
Vanessa et al. (2016).

In the present study in FGS, the tissue around both viable and dead $S$. haematobium eggs increased vascularity and a high density of macrophages, lymphocytes, foreign body giant cells, eosinophils, neutrophils, plasma cells, fibroblasts, and multinucleate histiocytes. Even calcified eggs induced the influx of immune cells and blood vessel proliferation (Kjetland et al, 2012). Egg deposition and granuloma formation eventually led to acute schistosomiasis followed by chronic inflammatory process associated with chronic infection (Elbaz and Esmat, 2013; Vanessa et al, 2016).

In the present study, egg deposition in the sub-epithelial and sub-mucosal layers with surrounding granulomatous reaction that was rich in macrophages and eosinophils as well as lymphocytes, plasma cells, foreign body giant cells and fibroblasts with variable degrees of fibrosis within the subepithelial and submucosal layers were detected in the $100 \%$ of FGS specimens.

Several cervical carcinoma associated with schistosomiasis were reported (Badawy, 1962; Youssef et al, 1970; Gwavava et al, 1984; Chenault and Hoang, 2006; Rachaneni et al, 2007; Toller et al, 2015; Kihara, 2015). But, others did not find association between cervical carcinoma and schistosomiasis (Williams, 1967; Coelho et al, 1979; Wright et al, 1982; Szela et al, 1993). In endemic areas, cervical schistosomiasis must be considered in the differential diagnosis of cancer (Swai et al, 2006). Fortunately, during the present study, none had malignant or premalignant cells.

Microscopic examination is the gold standard for schistosomiasis diagnosis (Feldmeier et al, 1999). In this study, the urogenital schistosomiasis in patients diagnosed by finding eggs using a standard urine filtration technique was $(18 / 200$ or $9 \%)$. This agreed with Khoby et al, (2000) in Egypt. However, others reported higher prevalence of urogenital schistosomiasis in endemic regions as Ogun State, Nigeria, was $47 \%$ 
(Ekpo et al, 2017), in South Africa was 30\% (Shukla et al, 2019).

The present results showed that 9/14 $(64.2 \%)$ genital schistosomiasis were positive for $S$. haematobium eggs in urine. This agreed with Vanessa et al. (2016). FGS can be associated with active egg-patent excretion, but also can present without eggs in urine. Several studies showed FGS women with $S$. haematobium eggs detected in urine (Kjetland et al, 1996; Leutscher et al, 1997; Poggensee et al, 1998). But, others found that up to $1 / 4$ of FGS cases were without eggs in urine (Shennan and Gelfand, 1971; Nouhou et al, 1998; Poggensee et al, 1998; Kjetland et al, 2008).

\section{Conclusion}

Female genital schistosomiasis was documented as not uncommon and exists in Sohag Governorate, Upper Egypt. In addition, the study observed that, the infection intensity of urogenital schistosomiasis influences the risk of having FGS. No specimens showed any malignant or premalignant cells. Female genital schistosomiasis affected entire female genital tract with serious health implications, so a specific action plan against FGS must be developed and organize an appropriate way not only to detect, but also to prevent.

\section{References}

Badawy, AH, 1962: Schistosomiasis of the cervix. Br. Med. J. 1:369-72.

Chenault, C, Hoang, MP, 2006: An unusual cervical finding: Female genital schistosomiasis with associated cervical severe squamous dysplasia (cervical intraepithelial neoplasia grade III). Arch. Pathol. Lab. Med. 130:e37-8.

Christinet, V, Lazdins-Helds, JK, Stothard, JR, Reinhard, RJ, 2016: Female genital schistosomiasis (FGS): From case reports to a call for concerted action against this neglected gynaecological disease. Inter. J. Parasitol.46:395-404.

Coelho, LH, Carvalho, G, Carvalho, JM, 1979: Carcinoma in situ and invasive squamous cell carcinoma associated with schistosomiasis of the uterine cervix a report of three cases. Acta Cytol. 23:45-8.

Colley, DG, Bustinduy AL, Secor WE, King CH, 2014: Human schistosomiasis. Lancet 383:
2253-64.

Culling, CFA, 1993: Handbook of Histological and Histochemical Techniques. $3^{\text {rd }}$ Ed., Butterworths, London, Boston.

Doehring, E, Vester U, Ehrich J, Feldmeier H, 1985: Circadian variation of ova secretion, proteinuria, hematuria and leukocyturia in urinary schistosomiasis. Kidney Int. 27: 667-71.

Downs, JA, Mguta C, Kaatano GM, et al. 2011: Urogenital schistosomiasis in women of reproductive age in Tanzania's Lake Victoria Region. Am. J. Trop. Med. Hyg. 84: 364-9.

Ekpo, U, Odeymi, Sam-wobo S, et al. 2017: Female genital schistosomiasis (FGS) in Ogun State, Nigeria: A pilot survey on genital symptoms and clinical findings. Parasitology 3, 10: e1-9.

Elbaz, T, Esmat, G, 2013: Hepatic and intestinal schistosomiasis: Review. J. Adv. Res. 4, 5: 445-52.

Feldmeier, H, Leutscher, P, Poggensee, G, Harms, 1999: Male genital schistosomiasis and haemospermia. Trop. Med. Intl. Hlth. 4:791-3.

Galappaththi-Arachchige, H, Hegertun, I, Holman, S et al, 2016: Association of urogenital symptoms with history of water contact in young women in areas endemic of S. haematobium: A cross-sectional study in rural South Africa. Int. J. Environ. Res Publ. Hlth. 13, 11135:112.

Gouveia, MJ, Brindley, PJ, Gartner, F, et al, 2019: Combination antihelmintic/ antioxidant against Schistosoma mansoni. Biomolecules 9: 54; doi: 10.3390/biom9020054.

Gwavava, NJ, Harrison, DJ, Gwata, TF, Zengeza, C, 1984: Incidence of schistosomiasis in surgical biopsies: a review of 284 cases. Cent. Afr. J. Med. 30:241-5.

Hegertun, I, Gundersen, K, Kleppa, E, et al, 2013: S. haematobium as a common cause of genital morbidity in girls: A cross-sectional study of children in South Africa. PLOs Neg. Trop. Dis. 13, 7:e20.

Helling-Giese, G, Aimee, Poggensee, D, 1996: Female genital schistosomiasis (FGS): relationship between gynecological and histopathological findings Acta Trop. 62, 257-67.

Hotez, PJ, Kamath, A, 2009: Neglected tropical diseases in sub-Saharan Africa: review of their prevalence, distribution, and disease burden. PLoS Negl. Trop. Dis. 3:e412.

Hotez, P, Whitham, M, 2014: Helminth infections: A new global women's health agenda. 
Obstet. Gynecol. 123:15-60.

Hotez, PJ, Harrison, W, Fenwick, A, et al, 2019: Female genital schistosomiasis and HIV/ AIDS: Reversing the neglect of girls and women. PLoS Negl. Trop. Dis 13, 4:e0007025. https: //doi.org/10.1371/journal.pntd.

Khoby, TE, Galal, N, Fenwick, A, et al, 2000: The epidemiology of schistosomiasis in Egypt summary findings in nine Governorates. Am. J. Trop. Med. Hyg. 62:88-99.

Kihara, JH, 2015: Female genital schistosomiasis: A neglected tropical disease infecting women of reproductive age in endemic areas. J. Infect. Dis. Diag. 1, 1:1-2.

Kjetland, EF, Poggensee, G, Helling-Giese, G, et al, 1996: Female genital schistosomiasis due to Schistosoma haematobium: Clinical and parasitological findings in women in rural Malawi. Acta Trop. 62:239-55.

Kjetland, EF, Ndhlovu, PD, Mduluza, T, et al, 2005: Simple clinical manifestations of genital Schistosoma haematobium infection in rural Zimbabwean women. Am. J. Trop. Med. Hyg 72: 311-9.

Kjetland, EF, Ndhlovu, PD, Gomo, E, et al, 2006: Association between genital schistosomiasis and HIV in rural Zimbabwean women. AIDS 20:593-600.

Kjetland, EF, Kurewa, EN, Ndhlovu, PD, 2008: Female genital schistosomiasis, a differential diagnosis to sexually transmitted disease: Genital itch and vaginal discharge as indicators of genital Schistosoma haematobium morbidity in a cross-sectional study in endemic rural Zimbabwe. Trop. Med. Inter. Hlth. 13, 1:509-17.

Kjetland, EF, Leutscher, PDC, Ndhlovu, PD, 2012: A review of female genital schistosomiasis. Trends Parasitol. 28:58-65.

Leutscher, P\, Raharisolo, C, Pecarrere, JL, et al, 1997: Schistosoma haematobium induced lesions in the female genital tract in a village in Madagascar. Acta Trop. 66:27-33.

Leutscher, P, Ravaoalimalala V, Raharisolo, C, 1998: Clinical findings in female genital schistosomiasis in Madagascar. Trop. Med. Int. Hlth. 3:327-32.

Madden, FC, 1899: A case of bilharziasis of the vagina. Lancet i:1716.

Mbabazi, PS, Andan, O, Fitzgerald, DW, Chitsulo, L, et al. 2011: Examining the relationship between urogenital schistosomiasis and HIV infection. PLoS Negl. Trop. Dis. 5:e1396.
Mbah, ML, Poolman, EM, Drain, PK, Coffee, MP, van der Werf, MJ, 2013: HIV and Schistosoma haematobium prevalence correlate in sub-Saharan Africa. Trop. Med. Int. Hlth. 18: $1174=9$.

Norseth, HM, Ndhlovu, PD, Kleppa, E, et al, 2014: The colposcopic atlas of schistosomiasis in the lower female genital tract based on studies in Malawi, Zimbabwe, Madagascar and South Africa. PLoS Negl. Trop. Dis. 8: e3229.

Nouhou, H, Seve, B, Idi, N, Moussa, F, 1998: Schistosomiasis of the female genital tract: Anatomoclinical and histopathological aspects: Apropos of 26 cases. Bull. Soc. Pathol. Exot. 91:221-3.

Poggensee, G, Kiwelu, I, Saria, M, et al, 1998: Schistosomiasis of the lower reproductive tract without egg excretion in urine. Am. J. Trop. Med. Hyg. 59:782-3.

Poggensee, G, Kiwelu I, Weger, V, et al, 2000: Female genital schistosomiasis of the lower genital tract: Prevalence and disease-associated morbidity in northern Tanzania. J. Infect. Dis. 181:1210-3.

Poggensee, G, Feldmeier, H, 2001: Female genital schistosomiasis: facts and hypotheses. Acta Trop. 79:193-210.

Rachaneni, S, Yaqoob, T, Spencer, C, 2007: Cervical schistosomiasis in an HIV positive patient with evidence of HPV infection. J. Obstet. Gynaecol. 27:632-3.

Renaud, G, Devidas, A, Develoux, M, et al, 1989: Prevalence of vaginal schistosomiasis caused by Schistosoma haematobium in an endemic village in Niger. Trans. R. Soc. Trop. Med. Hyg. 83:797-9.

Senghor, B, Aldiouma, D, Seydou, N, et al, 2014: Prevalence and intensity of urinary schistosomiasis among school children in the district of Niakhar, region of Fatick, Senegal. Parasit. Vect. 7:5-9.

Shennan, DW, Gelfand, M, 1971: Bilharzia ova in cervical smears a possible additional route for the passage of ova into water. Trans. R. Soc. Trop. Med. Hyg. 65:95-9.

Shukla, J, Elisabeth, K, Holmen, S, et al, 2019: Female genital schistosomiasis and reproductive tract infections. A cross-sectional study in rural adolescents in South Africa, med Rxiv posted online Oct. 18, doi: http:// dx.doi.org/ 10. 1101/19009233.

Steinmann, P, Keiser, J, Bos, R, et al, 2006: 
Schistosomiasis and water resources develop ment: Systematic review, meta-analysis, and estimates of people at risk. Lancet Infect. Dis. 6: 411-25.

Swai, b, Poggensee, G, Mtweve, S, Krantz, I, 2006: Female genital schistosomiasis as an evidence of a neglected cause for reproductive illhealth: A retrospective histopathological study from Tanzania. BMC Infect. Dis. 6, 1334:1-8. Szela, E, Bachicha, J, Miller, D, et al, 1993: Schistosomiasis and cervical cancer in Ghana. Int. J. Gynaecol. Obstet. 42:127-30.

Talaat, M, Watts, S, Mekheimar, S, et al, 2004: The social context of reproductive health in an Egyptian Hamlet: A pilot study to identify female genital schistosomiasis. J. Sci. Med. 58: 515-24.

Toller, A, Scopin, AC, Apfel, V, et al, 2015: An interesting finding in the uterine cervix: Schistosoma hematobium calcified eggs. Autops. Case Rept. 5:41-4.

Vanessa, C, Janis, K, Lazdins, J, et al, 2016: Female genital schistosomiasis (FGS): from case reports to a call for concerted action against this neglected gynecological disease. Inter. J. Parasitol. 46:395-404.

Vlassoff, C, 1997: The gender and tropical disease task force of TDR: achievements and challenges. Acta Trop. 67:173-80.

WHO, 2002: Prevention and control of schistosomiasis and soil-transmitted helminthiasis: Report of a WHO expert committee. Tech. Rep. Ser. 912:1-57.

WHO, 2015: Female Genital Schistosomiasis: a Pocket Atlas for Clinical Health Professionals. Geneva. ISBN: 9789241509299.

Williams, AO, 1967: Pathology of schistosomiasis of the uterine cervix due to $S$. haematobium. Am. J. Obstet. Gynecol. 98:784-91.

Wright, ED, Chiphangwi, J, Hutt, M, 1982: Schistosomiasis of female genital tract: A histopathological study of 176 cases from Malawi. Trans. R. Soc. Trop. Med. Hyg. 76:822-9.

Youssef, AF, Fayad, MM, Shafeek, MA, 1970: Bilharziasis of the cervix uteri. J. Obstet. Gynaecol. Br. Commonw. 77:847-51.

Fig. 1: Multiple degenerated and calcified bilharzial ova surrounded by bilharzial fibrotic reaction (fibrosis and few chronic inflammatory cells within sub-epithelial connective tissue of fallopian tube) (100x)

Fig. 2: Multiple degenerated and calcified bilharzial ova surrounded by bilharzial fibrotic reaction (fibrosis and few chronic inflammatory cells within sub-epithelial connective tissue of fallopian tube) (200x)

Fig. 3: Multiple degenerated and calcified bilharzial ova surrounded by bilharzial fibrotic reaction (fibrosis and few chronic inflammatory cells within sub-epithelial connective tissue of fallopian tube) (400x).

Fig. 4: Bilharzial granulomatous reaction of multiple degenerated bilharzial ova surrounded by dense chronic inflammatory cells of (lymphocytes, foreign body giant cells and numerous eosinophils) within sub-epthelial layer of fallopian tube (400x).

Fig. 5: Multiple calcified bilharzial ova surrounded by few chronic inflammatory cells of lymphocytes and eosinophils within submucosa of fallopian tube $(200 x)$

Fig. 6: Multiple calcified bilharzial ova surrounded by few chronic inflammatory cells of lymphocytes and eosinophils within submucosa of fallopian tube $(400 \mathrm{x})$

Fig. 7: Multiple degenerated and calcified bilharzial ova surrounded by chronic inflammatory cells of lymphocytes and eosinophils within sub-epithelial layers of the cervix (has stratified squamous epithelium). (400x)

Fig. 8: Multiple degenerated and calcified bilharzial ova surrounded by chronic inflammatory cells of lymphocytes and eosinophils within sub-epithelial layers of vagina (has stratified squamous epithelium). (200x) 


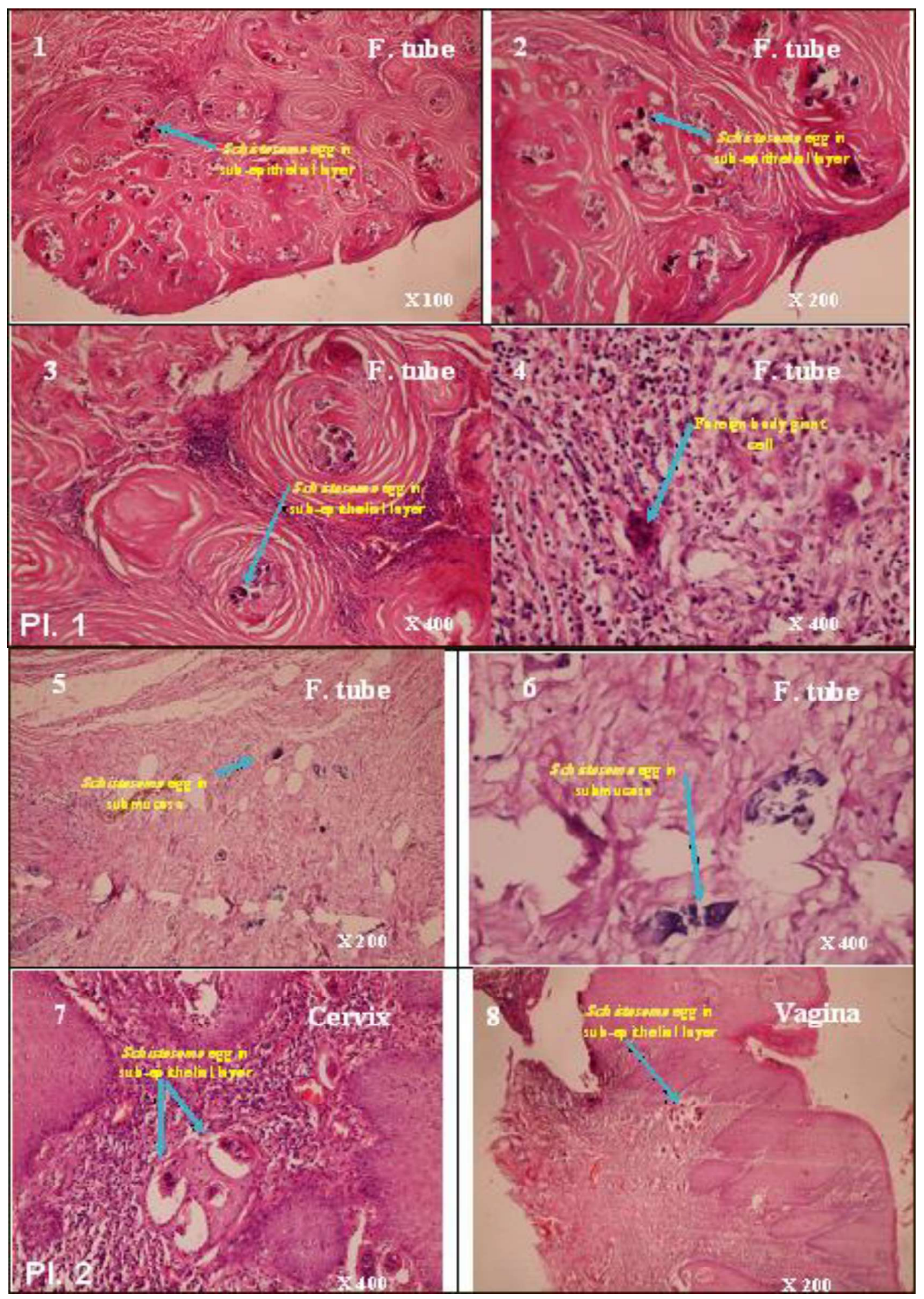

